\title{
\begin{tabular}{l|l} 
POLITIQUES \& & Politiques et management public
\end{tabular}
}

\section{Imitation et contextualisation dans la mise en œuvre des politiques publiques : le cas de la stratégie de l'État français en matière de propriété intellectuelle}

Imitation and Contextualization of Public Policies: the Case of French Policy of Intellectual Property

Mourad Attarça, Hervé Chomienne et Pascal Corbel

\section{OpenEdition} Journals

Édition électronique

URL : http://journals.openedition.org/pmp/5473

ISSN : 2119-4831

Éditeur

Institut de Management Public (IDPM)

Édition imprimée

Date de publication : 15 septembre 2012

Pagination : 473-491

ISBN : 978-2-7430-1437-7

ISSN : 0758-1726

Référence électronique

Mourad Attarça, Hervé Chomienne et Pascal Corbel, « Imitation et contextualisation dans la mise en œuvre des politiques publiques : le cas de la stratégie de l'État français en matière de propriété intellectuelle», Politiques et management public [En ligne], Vol 29/3 | 2012, mis en ligne le 12 février 2015, consulté le 30 avril 2019. URL : http://journals.openedition.org/pmp/5473 


\title{
Imitation et contextualisation dans la mise en œuvre des politiques publiques : le cas de la stratégie de l'État français en matière de propriété intellectuelle
}

\author{
Mourad Attarça ${ }^{a}{ }^{*}$, Hervé Chomienne $^{a}$ et Pascal Corbel ${ }^{b}$ \\ ${ }^{a}$ Maître de conférences en sciences de gestion \\ ${ }^{b}$ Maître de conférences HDR en sciences de gestion \\ Université de Versailles Saint-Quentin-en-Yvelines \\ Institut Supérieur de Management (ISM) \\ Laboratoire de recherche en Management LAREQUOI \\ 47, boulevard Vauban - 78047 Guyancourt Cedex
}

\section{Résumé}

Cet article s'intéresse aux politiques publiques en matière d'innovation et de propriété intellectuelle. Il vise à montrer qu'il existe, dans ce domaine, une forme de tension paradoxale entre la volonté des responsables publics d'imiter certains modèles d'inspiration nord-américaine et le souci d'adapter ces modèles aux spécificités du contexte institutionnel national. L'article s'appuie sur l'étude de la mise en place en 2011 d'un nouveau dispositif de valorisation des innovations dit « France Brevets ». La genèse et la mise en œuvre de ce dispositif reflètent I'hybridation croissante entre les secteurs publics et privés, mais aussi l'accélération de l'importation de solutions "génériques » à des problèmes publics d'un contexte national à l'autre. Même si elle demeure expérimentale, l'initiative «France Brevets » reflète une évolution du référentiel de l'action publique focalisée sur la recherche d'une performance mesurable. Cette initiative traduit également une démarche publique de type pragmatique : avec France Brevets, l'État se positionne comme accompagnateur -ou facilitateur- d'un «marché » de la recherche, publique et privée, en pleine mutation.

๑ 2012 IDMP/Lavoisier SAS. Tous droits réservés

Mots clés : politique publique, propriété intellectuelle, brevet, innovation, recherche publique.

*Auteur correspondant : mourad.attarca@uvsq.fr doi:10.3166/pmp.29.473-491 @ 2012 IDMP/Lavoisier SAS. Tous droits réservés 


\section{Abstract}

Imitation and Contextualization of Public Policies: the Case of French Policy of Intellectual Property. This article deals with public policies in innovation and in intellectual property. It aims to show that there exists a kind of paradoxical tension between the desire of public officials to imitate some american models seen as successful, and the desire to adapt these models to the specific national institutional context. The article is based on the study of the introduction in 2011 of a new organization called "France Brevets" (Patents France) in favor of innovation and public research. The launch and implementation of this organization reflect the increasing hybridization between the public and private sectors, but also the growing trend to import "generic » solutions to public problems from one national context to another. Although this organization is still experimental, it reflects an real evolution of the public action now focused on finding a measurable performance. This initiative also reflects a pragmatic approach in public policy: through "France Brevets", the French state seems to play a supporting role and to act as a facilitator in the changing market of public and private research which is changing.

(c) 2012 IDMP/Lavoisier SAS. Tous droits réservés

Keywords: public policy, intellectual property, patent, Innovation, public research.

\section{Introduction}

La crise que nous vivons actuellement est souvent réduite à son facteur déclencheur : la crise financière. Mais cette dernière ne doit pas masquer la transformation en profondeur que vit l'économie mondiale : une crise structurelle liée à la concurrence croissante que doivent affronter les pays industrialisés de longue date. Ainsi, face à la montée en puissance des pays émergents, d'abord sur des produits faiblement technologiques, puis de plus en plus complexes, les pays occidentaux n'ont guère d'autres choix que d'accentuer la différenciation de leurs produits. Leur capacité à engendrer des innovations technologiques, et notamment des innovations fondamentales s'appuyant sur leurs capacités scientifiques, est de ce point de vue déterminant. Dans des marchés fortement globalisés et pour beaucoup matures, la capacité d'innovation conditionne de plus en plus les perspectives de croissance, et donc le développement économique et social (Kegels, 2009).

S'agissant de la France, plusieurs rapports ont souligné les limites du système national en matière de transfert de technologie des institutions publiques de recherche vers l'industrie (Guillaume, 2007) ou dans la gestion de ces actifs immatériels (Lévy et Jouyet, 2006). Les autorités françaises se sont alors tournées vers les exemples de pays où le lien entre le monde académique était plus fort : à ce titre l'exemple des États-Unis vient en premier lieu. Depuis plusieurs années, des mesures ont été prises dans le but de dynamiser la coopération entre laboratoires publics. Nous pouvons ainsi citer : la création des pôles de compétitivité, dont l'inspiration principale est l'exemple de la Silicon Valley (Fligstein, 2001); le renforcement des objectifs de valorisation des institutions de recherche publique en instituant notamment une série d'indicateurs relatifs aux droits de propriété intellectuelle déposés et de leur valorisation, dont l'inspiration, même si on passe ici par d'autres canaux, est le 
Bayh-Dole Act; et, plus récemment, la mise en place d'un fonds d'investissement dans la propriété intellectuelle, "France Brevets », et des sociétés d'accélération du transfert de technologies (SATT) qui s'inspirent de la tendance enregistrée aux États-Unis à la création de fonds d'investissement privés dans la propriété intellectuelle.

Le but de cet article est de montrer qu'il existe une forme de tension paradoxale entre l'imitation de l'exemple nord-américain et son adaptation aux spécificités du contexte institutionnel français. Ce type de tension est courant en management ${ }^{1}$ et peut aussi être observé dans la mise en œuvre du New Public Management dans tous les pays de l'OCDE (Pollitt et Bouckaert, 2004; Emery et Giauque, 2008). Il s'agit en effet de trouver un point d'équilibre, non nécessairement stable, entre des forces antagonistes mais toutes deux indispensables. Nous nous appuierons pour cela sur l'analyse du nouveau dispositif de valorisation des innovations : «France Brevets ». La genèse et la mise en œuvre de ce dispositif reflètent l'hybridation croissante entre les secteurs publics et privés, mais aussi l'accélération de l'importation de solutions «génériques » à des problèmes publics, d'un contexte national à l'autre. En effet, si ce dispositif présente certaines caractéristiques qui trouvent leurs origines dans l'exemple nord-américain, ses modalités d'application restent spécifiques au «modèle français » de l'action publique et des évolutions qu'il enregistre (Muller, 2005). Nous montrerons ainsi que les tensions paradoxales provoquées par ces mutations et innovations institutionnelles sont exacerbées lorsque les fondements de l'exemple suivi sont très différents des valeurs de la sphère publique française. L'exemple nord-américain a en effet, dans ce cas, à la fois un rôle d'impulsion (c'est aux États-Unis que se sont développés les premiers marchés formalisés de droits de propriété intellectuelle et les premiers fonds de brevets) mais aussi de répulsion (éviter les dérives ${ }^{2}$ des marchés de propriété intellectuelle).

Cet article est structuré en trois parties. Nous commencerons par présenter le contexte des politiques françaises en matière de propriété intellectuelle et leur mise en œuvre et nous montrerons en quoi ils font émerger une tension imitation/adaptation. Nous analyserons ensuite l'une des manifestations les plus récentes de ces politiques : la genèse et la mise en place du fond « France Brevets ». Nous nous appuierons pour cela sur une étude empirique menée sous forme d'entretiens avec des acteurs impliqués dans sa création. Nous terminerons par une discussion sur le positionnement de ce dispositif dans la politique publique française de recherche et d'innovation en analysant particulièrement les tensions et effets paradoxaux qu'ils pourraient provoquer.

\section{Les évolutions récentes de la stratégie des pouvoirs publics français en matière de propriété intellectuelle : une adaptation de l'exemple nord-américain?}

La propriété intellectuelle a longtemps été un domaine plutôt négligé par les pouvoirs publics français. Plusieurs rapports (Lévy et Jouyet, 2006; Guillaume, 2007) ont souligné les faiblesses du secteur public français en matière de gestion du patrimoine immatériel d'une part, et de transfert des technologies innovantes vers l'industrie d'autre part. Depuis le début

\footnotetext{
${ }^{1}$ On peut la rapprocher du couple ago-antagoniste « hétérogénéisation » / « homogénéisation » relevé par Martinet (1990).

${ }^{2}$ Comme nous le verrons plus loin, les sociétés dites «patent troll» figurent parmi ces dérives. II s'agit d'entreprises dont le modèle économique consiste à acquérir des brevets et à utiliser systématiquement la menace de litiges pour tenter d'obtenir d'importantes royalties de la part des contrefacteurs potentiels.
} 
des années 2000, et l'adoption des « objectifs de Lisbonne » qui prévoient un accroissement des investissements en R\&D à un niveau minimum de $3 \%$ du PIB, la propriété intellectuelle semble être devenue un axe important des politiques publiques en faveur de l'innovation. Plusieurs outils stratégiques ont ainsi été mis en place visant à combler l'écart avec les pays les plus performants dans ce domaine, et notamment avec les États-Unis : la mise en place des pôles de compétitivité, la formalisation des objectifs en matière de valorisation de la recherche publique, et la mise en place des Sociétés d'Accélération de Transfert Technologique. Nous nous concentrerons sur les deux derniers, plus spécifiquement liés à la propriété intellectuelle.

\subsection{Le renforcement des objectifs des organisations publiques de recherche en matière de valorisation}

La loi organique relative aux lois de finances (LOLF), adoptée en 2001, a été l'occasion de formaliser et de stabiliser les objectifs des organismes publics de recherche et des universités en matière de valorisation de la recherche dans le cadre d'une stratégie nationale. Les établissements publics de recherche ont été incités à élaborer des plans annuels de performance sur la base d'indicateurs (comme la part des ressources apportées aux opérateurs par les redevances sur titre de propriété intellectuelle ou la part des contrats de recherche passés avec des entreprises dans les ressources des opérateurs), et leurs budgets ont été plus directement reliés à l'atteinte des objectifs. En 2009, les pouvoirs publics élaborent un plan en matière de recherche et d'innovation qui fixe des priorités stratégiques et constitue un cadre général dans lequel viennent s'insérer l'ensemble des dispositifs en lien avec la politique de recherche et d'innovation française ${ }^{3}$. Le plan vise notamment à encourager la mise en place de structures de valorisation de la recherche publique.

Cette approche reprend la logique mise en œuvre aux États-Unis dès le début des années 1980 avec le Bayh-Dole Act. Cette loi visait à clarifier les questions de propriété industrielle dans le cas des projets de recherche financés par des fonds publics fédéraux. Elle confère notamment la propriété des inventions aux universités et leur donne le droit de les licencier, sur une base exclusive ou non. Le Bayh-Dole Act a permis d'accélérer le développement, au sein des universités, de structures de valorisation. Durant les années 80 et 90, les dépôts de brevets et les revenus de licences des universités connurent ainsi une augmentation considérable (Mowery et al., 2001).

Ces structures de valorisation, qui prennent des formes diverses ${ }^{4}$, ont dû gagner leur légitimité au sein d'institutions qui n'avaient pas développé une culture de la propriété intellectuelle; une culture qui s'oppose partiellement à la logique de l'open science, dominante dans les institutions de recherche académique (Dasgupta et David, 1994). Elles se sont constituées assez rapidement, en majorité après 1999 (à l'exception de quelques grands organismes de recherche publique comme le CEA qui avaient développé ce type d'activité bien avant), et avec des ressources limitées ${ }^{5}$. Elles se sont donc construites sur une base de

\footnotetext{
${ }^{3}$ Ministère de l'enseignement supérieur et de la recherche, Stratégie nationale de recherche et d'innovation, Rapport général, 2009 (www.recherche.gouv.fr).

${ }^{4}$ D'après une étude menée en 2008 par le Bureau d'Économie Théorique et Appliquée (Bach et al., 2008) près des deux tiers sont des départements internes, près d'un quart des SAIC (Service des Activités Industrielles et Commerciales), $6 \%$ des filiales majoritaires et $3 \%$ des associations.

${ }^{5}$ D'après l'étude de Bach et al. (2008), la moitié de ces structures ont un budget moyen inférieur à 300 k€/an et des effectifs inférieurs à 3,5 équivalent-temps plein.
} 
compétences assez fragile et ont dû s’imposer face à des industriels plus aguerris et parfois surpris du changement de politique assez radical des organismes publics de recherche et des universités en matière de propriété intellectuelle. Les structures de valorisation ont ainsi été souvent perçues par les industriels comme étant des obstacles aux relations avec les laboratoires publics, alors que leur rôle revendiqué est justement de faciliter ces relations (Corbel et al., 2011). Même si ces difficultés peuvent être progressivement levées au fil des coopérations et des échanges entre partenaires, elles restent en partie liées à la nature même des relations entre acteurs publics et acteurs privés qui poursuivent des finalités différentes

Aux États-Unis, le Bayh-Dole Act a été mise en œuvre dans un contexte où les relations entre universités et industries s'inscrivaient dans une longue tradition de coopération qui remontait aux années 70 (Mowery et al., 2001). Le renforcement des activités de valorisation a donc été progressif. En France, à l'inverse, hormis dans quelques organismes comme le CEA, la culture de la propriété intellectuelle et de la valorisation de la recherche publique est beaucoup moins développée. La priorité donnée à ces questions a émergé brutalement, sous la pression de l'État, sans vraiment laisser le temps aux institutions publiques de recherche de construire les compétences et de mettre en place les routines organisationnelles permettant de gérer cette transition.

\subsection{La mise en place des Sociétés d'Accélération de Transfert Technologique (SATT)}

Malgré la dynamique initiée dans les années 2000, le système français de valorisation de la recherche publique est considéré comme peu efficace car trop fragmenté, sous capitalisé et faiblement doté en ressources humaines ${ }^{6}$. Dans leur rapport sur les priorités stratégiques et le grand emprunt national, Alain Juppé et Michel Rocard ${ }^{7}$ soulignent que « la France souffre en outre d'une insuffisante culture de la valorisation des résultats de sa recherche et de la trop faible place accordée à la recherche partenariale public-privé. Les pays les plus dynamiques savent déposer des brevets en nombre important et réunir des compétences interdisciplinaires de haut niveau sur un même site, en alliant enseignement supérieur, recherche et développement industriel» (p. 28). Dans le même rapport, les auteurs proposent ainsi la constitution d'un «fonds national de valorisation des projets innovants, doté en capital d'un milliard d'euros. Ce fonds aurait pour objet de protéger et valoriser les résultats de la recherche publique, ainsi que d'accélérer les transferts de technologie entre la recherche fondamentale et les entreprises » (p. 70).

Dans le cadre de la mise en œuvre du rapport Juppé-Rocard (programme « Investissements d'Avenir »), l'État a effectivement décidé la création de Sociétés d'Accélération de Transfert Technologique (SATT), dotées d'une enveloppe d'un milliard d'euros ${ }^{8}$. L'objectif des SATT est triple 9 : d'une part, rendre plus lisible le dispositif de valorisation de la recherche auprès des acteurs concernés (logique du guichet unique); d'autre part, avoir un rôle de

\footnotetext{
${ }^{6}$ Selon Vincent Lamande, ancien Président du Réseau C.U.R.I.E pour la valorisation de la Recherche publique, en juin 2010, il existait en France 163 structures de valorisation. Source : « La création des SATT constitue un moment historique pour la valorisation! », réseau ACIES Consulting Group, (www.acies-cg.com).

${ }^{7}$ Rocard M, Juppé A., (2009), « Investir pour l'avenir : priorités stratégiques d'investissement et emprunt national », La Documentation française, novembre 2009, 128 p..

${ }^{8}$ En fait la dotation n'est que de 950 millions d'euros, 50 millions d'euros étant consacrés au projet France Brevet.

${ }^{9}$ Selon Ronan Stéphan, Directeur Général de la Recherche et de l'Innovation au ministère de la Recherche et de l'Enseignement Supérieur.
} 
mise en réseau des différentes institutions de la recherche publique dans un territoire donné (universités, écoles d'ingénieurs, organismes nationaux) durant la phase de maturation des innovations; enfin, favoriser le rapprochement des acteurs publics de la recherche avec les acteurs industriels et les pôles de compétitivité, avec une exigence de retour sur investissement (transfert technologique vers des entreprises ou création d'entreprises).

Les $\mathrm{SATT}^{10}$, dont l'actionnariat est partagé entre l'État (qui détient 1/3 du capital via la Caisse des Dépôts et Consignations) et les membres fondateurs (universités ou Pôles de Recherche et d'Enseignement Supérieur), ont un objectif d'équilibre financier au bout de 10 ans. Si les SATT ont comme finalité la valorisation de l'innovation, les droits de propriété intellectuelle restent la propriété des établissements de recherche. Les structures des SATT pourront s'appuyer sur d'autres dispositifs mis en place par l'État dans le cadre du programme Investissements d'Avenir : le Fonds national d'amorçage doté de 400 millions d'euros et pouvant financer une vingtaine de fonds d'amorçage et le dispositif France Brevets dont nous allons étudier dans ce qui suit les conditions d'émergence et de mise en œuvre.

\section{France Brevets : genèse et contours d'un dispositif de politique publique entre État et Marché}

L'exemple de la création de France Brevets nous semble particulièrement intéressant pour étudier les tensions résultant de l'adaptation au contexte français de dispositifs empruntés à l'étranger. En effet, il s'agit d'une entité qui s'inspire pour une part d'organisations purement financières, les patent trolls. Ces entreprises, qui se sont développées aux États-Unis au début des années 2000 (Pénin, 2010), acquièrent des portefeuilles de brevets sous-valorisés puis tentent de les bonifier en s'attaquant juridiquement aux entreprises potentiellement contrefactrices. Sous la menace de procès, les patent trolls obtiennent des royalties beaucoup plus élevées que celles qu'elles auraient obtenues dans une négociation classique (Reitzig et al., 2007). Le comportement des patents trolls, qui détournent la finalité de la réglementation américaine très protectrice de la propriété intellectuelle, pour agir en prédateur, a fait l'objet de sévères critiques (McCurdy, 2008; Pénin, 2010 ; Reitzig et al., 2007). Les effets induits par leurs pratiques ont même abouti à une modification substantielle de la jurisprudence concernant les contrefaçons de brevets (Diessel, 2007).

Il est manifeste, au regard des objectifs attribués à France Brevets ${ }^{11}$, que cette structure publique d'investissement ne devrait pas reprendre les méthodes agressives de négociation de ses « homologues » nord-américaines. C'est néanmoins une organisation qui s’appuie sur une croyance forte dans le développement des marchés de brevets, mis en

\footnotetext{
10 L'appel à projet SATT de 2010 a permis de retenir en juin 2011 cinq SATT : Université de Strasbourg, Université Pierre et Marie Curie, PRES Université de Toulouse, PRES Université Paris Cité, Université de la Méditerranée.

${ }^{11}$ Convention du 2 septembre 2010 entre l'État, I'Agence nationale de la recherche et la Caisse des Dépôts et Consignations relative au programme d'investissement d'avenir (action « France Brevets »), Journal officiel de la République française, Textes généraux, 4 septembre 2010. Ainsi que déclaration du Président de la République en conclusion des États Généraux de l'Industrie à Marignane le 4 septembre 2010 relative à la création du fonds d'investissement France Brevets (www.elysee.fr).
} 
exergue par des travaux récents du Centre d'Analyse Stratégique (Lallement, 2009) ${ }^{12}$ et du Conseil d'Analyse Économique (Guellec et al., 2010) alors même qu'il s'agit d'actifs dont les caractéristiques sont en contradiction avec les conditions facilitant l'efficience de ces derniers (Teece, 1998).

Nous sommes donc en présence d'un exemple issu du monde financier et fondé sur une logique de développement de la sphère des marchés, qui va être mis en œuvre par la Caisse des Dépôts et Consignations (CDC), sous une forme centralisée et avec un contrôle direct de l'État. Il en résulte la juxtaposition de buts fondés sur l'intérêt général (valorisation d'inventions issues de la sphère publique ou de PME) et de contraintes de rentabilité qui peuvent susciter un doute sur le modèle économique de cette nouvelle institution et sur la capacité de cette greffe à s'insérer dans un système français de recherche et d'innovation connaissant depuis quelques années une succession de réformes institutionnelles majeures (Lesourne et Randet, 2010).

Ce cas présente également une caractéristique qui pourrait être considérée comme une limite mais qui constitue pour nous une qualité supplémentaire : sa création est très récente. La structure France Brevets a été initiée dans une logique d'expérimentation dans un domaine où il existe peu de repères. On peut donc s'attendre à ce que la stratégie de la structure soit peu stabilisée au cours des premières années. Une étude menée dans quelques années aurait alors à discerner entre ce qui relève de la logique d'origine et ce qui relève d'une rationalisation a posteriori. En menant notre étude à la naissance du projet, nous pouvons donc mieux appréhender sa logique fondamentale même s'il a déjà connu quelques réorientations, avant même la création de la structure.

Pour essayer de comprendre les différentes dimensions de la logique du projet, les appuis et résistances qu'il a pu rencontrer, ainsi que son modèle d'affaires, nous avons mené une série de six entretiens ${ }^{13}$ avec des acteurs ayant été impliqués directement ou indirectement dans le projet :

- l'initiateur du projet France Brevets : Patrick Terroir ${ }^{14}$;

- des conseils en propriété intellectuelle ayant participé au processus d'émergence du dispositif France Brevets : Christian Nguyen van Tran ${ }^{15}$, Dominique Deberdt ${ }^{16}$ et Patrick Pierre ${ }^{17}$;

- des conseils en propriété intellectuelle ayant suivi en tant qu'experts l'émergence du dispositif France Brevets : Pierre Breesé ${ }^{18}$ et Jean-Claude Prager ${ }^{19}$.

Les entretiens, de type semi-directif, ont permis d'aborder trois grands thèmes : la genèse du projet, son processus de mise en œuvre et le modèle économique de France Brevets.

\footnotetext{
${ }^{12}$ Lallement R., «Marchés du savoir et transactions fondées sur la propriété intellectuelle. Interrogations d'ordre conceptuel, éléments de cadrage statistique et quelques expériences étrangères », Compte rendu du groupe de travail « Développement des marchés de la connaissance » organisé par le Conseil d'Analyse Économique et l'OCDE, séance du 27 novembre 2009 au Centre d'analyse stratégique.

${ }^{13}$ Entretiens semi-directifs, d'une durée variant de $1 \mathrm{~h}$ à $2 \mathrm{~h} 30$.

${ }^{14}$ Ancien Directeur régional de la Caisse des dépôts pour Paris-lle-de-France, ancien délégué aux pôles de compétitivité et à l'innovation et, depuis janvier 2011, directeur général délégué de CDC Propriété intellectuelle.

${ }^{15}$ Conseil en Propriété Industrielle.

${ }^{16}$ En charge, de 1996 à 2000, des questions de propriété industrielle au Ministère de l'Économie, des Finances et de l'Industrie. Responsable, de 2001 à 2010, de l'Observatoire de la Propriété Intellectuelle.

${ }^{17}$ Conseil en Propriété Industrielle.

${ }^{18}$ Conseil en Propriété Industrielle.

${ }^{19}$ Expert en politique publique d'innovation. Co-rédacteur d'un rapport pour le Conseil d'études économiques : Guellec et al. (2010), Les marchés de brevets dans l'économie de la connaissance, Rapport pour le Conseil d'Analyse, 255 p.
} 


\subsection{La genèse du projet}

Il apparaît tout d'abord que le projet émane directement de la Caisse des Dépôts et Consignations (CDC) qui a commencé à s'intéresser à l'innovation à partir de 2004-2005, principalement à travers des actions dans le domaine du numérique, des nouvelles énergies et des pôles de compétitivité. C'est à partir de 2008 que la CDC commence formellement à s'intéresser au monde de la propriété intellectuelle et notamment à celui des brevets. Ses projets d'intervention se sont fondés sur deux constats : l'état de la valorisation de la recherche publique, considéré comme préoccupant, et le décalage entre l'importance de la connaissance dans l'économie d'aujourd'hui et de demain et le fonctionnement des marchés de technologie, jugé opaque et dirigé davantage vers le litige que vers l'échange. Ces réflexions s'inscrivent par ailleurs dans un contexte de mutation de l'économie de la propriété intellectuelle, avec notamment l'apparition aux États-Unis de nouveaux acteurs, des intermédiaires, dans la chaîne de valorisation des brevets : brokers, plateformes électroniques, pools de brevets, fonds de brevets, patent trolls, places d'enchères, offices de transferts technologiques (Guellec et al., 2010).

Découvrant le monde de la propriété intellectuelle et ses enjeux, la CDC va se tourner vers des experts français (l'Institut National de la Propriété Intellectuelle, le Conseil d'Analyse Économique) mais aussi vers certains acteurs majeurs des marchés de brevets aux États-Unis comme l'entreprise Ocean Tomo ${ }^{20}$. Elle va chercher à se positionner en pionnier et leader européen sur ces questions en élaborant «les infrastructures d'une nouvelle économie de la propriété intellectuelle » (initiateur du projet). Le but pour la CDC est de développer un ensemble d'outils autour de l'enjeu de la valorisation, formant un système : outils d'évaluation, places de marché, fonds d'investissement. En 2010, un rapport du Conseil d'Analyse Économique (Guellec et al., 2010) aborde les enjeux des marchés des brevets dans l'économie de la connaissance et développe les orientations souhaitables d'une politique publique dans ce domaine.

C'est dans ce cadre que se situe le projet « France Brevets », pensé à l'origine comme un fonds d'investissement dans le marché des brevets. Le projet est présenté aux pouvoirs publics français ainsi qu'à la Commission européenne. Des réflexions sont alors menées sur un fonds européen mais les pouvoirs publics français choisissent de lancer la version française à titre expérimental sans attendre un éventuel accord au niveau européen .

Le dispositif « France Brevets » est ainsi prévu dans le programme «Investissements d'Avenir », issu du rapport Juppé-Rocard ${ }^{21}$ remis au Président de la république en 2009. Ce dispositif, complémentaire aux pôles de compétitivité, vise notamment à améliorer la valorisation des recherches issues des pôles de compétitivité. Il doit également faciliter les relations entre chercheurs, structures de valorisation et industriels ${ }^{22}$ et constituer

\footnotetext{
${ }^{20}$ Ocean Tomo est une entreprise américaine qui a mis en place les premières places d'enchères dédiées au marché des brevets. Cette entreprise annonce avoir réalisé près de 100 millions de dollars de transactions depuis 2006. Ocean Tomo a élaboré un modèle d'évaluation des brevets en fonction de leur potentiel de marché : modèle IPQ ou Intellectual Property Quotient (Guellec et al., 2010 : 41).

${ }^{21}$ Rocard M, Juppé A., op. cit.

22 «France Brevet » est présentée comme un « véritable guichet unique de proximité pour améliorer le service rendu aux chercheurs et aux entreprises » : document du ministère de l'enseignement supérieur et de la recherche, "La valorisation de la recherche au cœur des investissements d'avenir : le fonds national de valorisation », disponible sur www.enseignementsup-recherche.gouv.fr.
} 
« une véritable passerelle publique-privé [en matière de gestion de portefeuilles de brevets] pour soutenir l'économie ${ }^{23} »$.

\subsection{Le processus de lancement de France Brevets}

France Brevets a été officiellement créée le 31 mars 2011, sous forme d'une Société par Action Simplifiée dotée d'un capital de 100 millions d'euros dont 50 millions en provenance de la CDC et 50 millions en provenance de l'État au travers du programme d'investissement d'avenir.

Les objectifs et le cadre d'action de la structure sont fixés dans une convention dans laquelle l'Agence Nationale de la Recherche (ANR) est également partie prenante. Une période expérimentale est prévue afin de valider les hypothèses de construction du modèle économique de France Brevets mais aussi pour affiner le positionnement et le modèle économique ${ }^{24}$, notamment les secteurs technologiques cibles. Plusieurs autres dispositions témoignent du caractère novateur de la démarche sur le plan de l'action publique : les dirigeants de cette entité sont issus du secteur privé et reconnus pour leurs compétences et leur expérience de la valorisation de la recherche et des fonds d'investissement; des processus de pilotage et d'évaluation sont formellement prévus dans la convention portant création de France Brevets; l'État et la CDC se fixent un objectif de retour sur investissement de $8 \%$. La structure France Brevets relève ainsi d'une démarche managériale qui semble cohérente sur le plan formel et qui recherche autant l'efficience financière que l'efficacité au service de la politique de recherche et d'innovation de l'État.

Le principe de départ de France Brevets est de permettre la valorisation à grande échelle des brevets issus de la recherche publique. En principe, une telle structure permet de répondre à deux des problèmes liés à la fragmentation du système de valorisation de la recherche publique : d'un côté, celui de la compétence, que nous avons déjà évoqué : une telle structure est davantage susceptible de réunir l'ensemble des compétences nécessaires pour évaluer le potentiel des technologies protégées, défendre les droits et négocier des licences, notamment au niveau international; de l'autre, elle aura la capacité de rassembler des ensembles («clusters ») de brevets formant un système ayant beaucoup plus de valeur ensemble que séparément.

L'accueil de la part des acteurs de la recherche publique a toutefois été plutôt prudent. D'une part, selon l'un de nos interlocuteurs (expert en propriété intellectuelle), le langage très « économique » utilisé à l'origine du projet ( « place de marché », " marché de brevets », « enchères de brevets ») a pu désarçonner certains organismes de recherche peu habitués à ce rapport à la connaissance : «Il y a eu une levée de boucliers dans le

\footnotetext{
${ }^{23}$ Discours de Valérie Pécresse, ministre de l'enseignement supérieur et de la recherche, à l'occasion de la clôture de cérémonie de signature par France Brevets des conventions de partenariat avec l'Institut National de Propriété Industrielle (INPI), OSEO et I'Institut Télécom le 9 juin 2011.

${ }^{24}$ Pendant une période d'au plus douze mois, l'État et la Caisse des dépôts fixent à France Brevets l'objectif de procéder à la validation des principales hypothèses retenues pour procéder à sa création. À cette fin, France Brevets réunit tous les éléments utiles pour valider la pertinence de sa stratégie : « (...) Sous douze mois : conduire des études de marchés sur le potentiel de licensing de 2 à 3 secteurs prioritaires; évaluer les conditions pour réaliser des grappes de brevets et conduire une politique de licensing; définir une stratégie détaillée du fonds; évaluer les moyens nécessaires pour poursuivre la stratégie adoptée; élaborer le plan d'affaires initial de France Brevets en fonction de ces éléments; proposer les objectifs et indicateurs opérationnels d'activité pour la phase d'exploitation suivant la phase de validation » (extrait de la convention État-CDC-ANR).
} 
microcosme sur le thème : on est en train de créer une bulle, il ne peut pas y avoir de marché sans création de valeur ». D'autre part, plusieurs de nos interlocuteurs (conseils et experts en propriété intellectuelle) ont admis que la manière dont le projet a été présenté n'a pas facilité sa réception puisque l'argumentation reposait sur les faibles performances des structures de valorisation existantes.

Nos interlocuteurs nuancent toutefois ce constat, soit en indiquant que les structures de valorisation des organismes publics de recherche se montrent désormais plus réceptives qu'au moment de la présentation initiale du projet, soit en précisant que les réticences se situaient plus au niveau des grands organismes qu'au niveau des universités, davantage séduites. Les échanges avec les industriels et cet accueil mitigé, sachant que la CDC veut s'appliquer à elle-même une logique de marché et donc ne rien imposer, ont conduit à élargir quelque peu le concept d'origine et à intégrer la valorisation de brevets d'entreprises, notamment des PME qui n'ont pas, elles non plus, les moyens de mener une politique de valorisation active. Une première transaction a ainsi concerné une entreprise en liquidation.

Le projet semble donc actuellement cohérent : d'une part, il permet à des structures ayant des ressources limitées, d'espérer valoriser leurs brevets à une échelle difficile à atteindre pour elles; d'autre part, il favorise l'essor de PME en leur permettant d'asseoir leur développement sur des technologies exclusives ou d'assurer leur liberté d'exploitation.

\subsection{Les limites de la référence au modèle nord-américain}

L'exemple du système nord-américain a joué un grand rôle dans la mise en œuvre du projet France Brevets. Il sert d'abord de référence implicite pour un modèle performant de gestion de la valorisation de la recherche (même si d'autres références comme le modèle allemand sont aussi considérées). Ensuite, et surtout, lorsque l'on parle de marchés de brevets et de fonds d'investissement en propriété intellectuelle, c'est immédiatement aux États-Unis que l'on pense. Comme le soulignent Guellec et al. (2010: 41), $72 \%$ des nouveaux acteurs « accélérateurs d'innovation » (fonds de brevets, pools de brevet, brokers...) apparus ces dix dernières années sont localisés aux États-Unis.

Combinant une législation et une jurisprudence très favorables aux détenteurs de brevets, qui explique partiellement une forte hausse des dépôts de brevets au cours des années 1990 (Kortum et Lerner, 1999) et une forte financiarisation de son économie, les États-Unis réunissaient toutes les conditions pour voir émerger une forme d'industrie de la propriété intellectuelle. Ainsi, de nombreux acteurs de natures différentes se sont développés sur ce marché : des entreprises visant à organiser des places de marché, à l'origine à travers des ventes aux enchères et à fournir des outils d'analyse (Ocean Tomo), des fonds d'investissement (dont le principal est Intellectual Ventures ${ }^{25}$ ), ou encore des patent trolls (cf. supra). L'ensemble donne l'impression que, même si c'est avec des excès (les agis-

\footnotetext{
${ }^{25}$ Intellectual Venture (IV) est une entreprise qui a été créée en 2000 par Nathan Myhrvold, ancien Chief Technology Officer de Microsoft. IV possède un portefeuille de brevets très large, dans des secteurs aussi variés que les technologies de l'information et la communication, l'instrumentation médicale ou les technologies de l'énergie nucléaire. IV se présente également comme une entreprise de R\&D : 1000 brevets ont été directement déposés par Intellectual Venture.
} 
sements des patent trolls) et des échecs (les ventes aux enchères), un marché des technologies se structure progressivement aux États-Unis et que l'Europe prend du retard.

Pour ses initiateurs, le projet France Brevets devait permettre à la France de s'inscrire dans cette dynamique et de ne pas rater les éventuelles opportunités des nouveaux marchés des brevets, d'autant que plusieurs pays asiatiques (Japon, Chine, Corée du Sud, Taïwan) se sont également engagés dans la création de fonds d'investissement dans les brevets. De plus, le caractère très agressif de certaines de ces sociétés financières incite à mettre en place des structures différentes en Europe pour anticiper leur arrivée sur le marché français des brevets. En effet, si la fragmentation du système européen de la propriété intellectuelle (qui reste fondamentalement national) rend pour l'instant moins intéressante l'utilisation de telles méthodes en Europe, qu'en serait-il si une juridiction européenne était effectivement mise en place? L'accélération du processus de mise en place d'un brevet unitaire européen ${ }^{26}$ rend ce risque très probable. En effet, l'harmonisation des réglementations de la propriété intellectuelle favorisera l'émergence d'un marché européen des brevets et, vraisemblablement, le développement de structures d'investissements dans les brevets à l'échelle de l'Union Européenne.

Dès lors, il n'est pas étonnant que les exemples nord-américains reviennent assez abondamment dans le discours de nos interlocuteurs. Ocean Tomo a, semble-t-il, joué un rôle important au début du projet, l'un de ses volets consistant à essayer d'adapter au contexte européen les outils de «scoring » de brevets mis au point par cette entreprise. La mise au point de ce type d'outil est d'ailleurs l'un des piliers du projet France Brevets puisque ce dernier repose assez largement sur l'idée de l'application de méthodes systématiques d'évaluation d'un grand nombre de brevets tranchant avec le caractère artisanal des transactions d'aujourd'hui. France Brevets doit pouvoir éviter un biais défavorable dans les brevets qui lui sont confiés : il serait en effet tentant pour les structures de valorisation de ne lui confier que des brevets sans grande valeur. L'utilisation de ce type d'outil permet de réaliser une évaluation « en masse » d'un portefeuille de brevets et peut donc en principe d'atténuer ce risque.

France Brevets est aussi en contact avec l'entreprise américaine Intellectual Ventures dont le modèle d'affaire est celui du « capital-invention », c'est-à-dire celui d'un capital-risqueur appliqué au marché des brevets ${ }^{27}$. Le modèle de Intellectual Venture comprend une forte dimension de valorisation, incluant notamment, mais pas exclusivement, des partenariats avec des universités et des centres de recherches publics nord-américains.

L'adaptation du modèle nord-américain au contexte français pose toutefois un premier problème : celui de l'adaptation des moyens. La plupart des entreprises américaines évoluant dans le «marché des brevets » ont levé plusieurs centaines de millions de dollars. La plus richement dotée, Intellectual Ventures, possède un portefeuille estimé à 30000 familles de brevets représentant un actif immatériel évalué à près de 5 milliards de dollars. De son côté, France Brevets, avec une capitalisation de 100 millions d'euros, devrait pouvoir réunir 4000 familles de brevets, bien que l'objectif de l'État soit d' «au moins 10000 familles

${ }^{26}$ Initié en 2010, le projet de «juridiction unifiée relative au brevet» a été publié en mars 2011. En juin 2011, une orientation générale du Conseil de I'Union Européenne a adopté le principe d'un brevet unitaire qui s'appliquera dans l'ensemble des pays ayant adhéré au processus, aux côtés des brevets nationaux. Source : Office Européen des Brevets.

${ }^{27}$ Voir : Nathan Myhrvold, "The Big Idea: Funding Eureka!", Harvard Business Review, March 2010. 
de brevets $»^{28}$. Cela serait dans doute suffisant, en termes d'impact industriel, si le fond se concentrait sur un domaine technologique particulier, comme celui des technologies de l'information. Cela risque d'être plus difficile si les fonds sont répartis dans un grand nombre de domaines ${ }^{29}$. En effet, plusieurs secteurs devraient faire partie du périmètre d'action de France Brevets : prioritairement, les technologies de l'information et de la communication, mais aussi l'aéronautique et l'espace, la chimie et les matériaux ${ }^{30}$. Or, si les fonds investis par les pouvoirs publics ont permis d'augmenter l'enveloppe globale de France Brevets, l'introduction d'une logique politique (par exemple la nécessité de sauvegarder des emplois ou de soutenir certaines entreprises en particulier) pourrait conduire à une dispersion des ressources. Dès lors, France Brevets a dû quelque peu adapter son modèle d'affaires. Le fonds devrait privilégier les prises de mandats de valorisation de brevets plutôt que leur acquisition. Cela permet de réduire les coûts mais risque parfois de compliquer les transactions.

La deuxième difficulté nous semble être beaucoup plus importante. La valorisation des brevets n'est possible que si l'on se montre prêt à défendre ses droits en cas de contrefaçon. Or, de ce point de vue, France Brevets devra composer avec un exemple nord-américain qui joue clairement le rôle de répulsif : celui des patent trolls qui est considéré comme un phénomène nuisible à l'innovation et à l'essor des entreprises innovantes (Pénin, 2010; Reitzig et al., 2007). Dans le contexte français, il est évidemment inimaginable, pour une société créée au nom de l'intérêt général, de pouvoir être assimilée à un patent troll. Cela exclut l'utilisation de techniques « agressives » de valorisation mais rend également difficile l'engagement d'un procès contre un contrefacteur, qui risque pourtant d'être indispensable un jour ou l'autre. Intellectual Ventures, qui a toujours voulu se différencier des patent trolls n'en a pas moins lancé une série d'actions en contrefaçon aux États-Unis ${ }^{31}$. Or, n'oublions pas que, si France Brevets est une structure semi-étatique et $100 \%$ publique, la convention régissant sa création fixe un objectif de rentabilité de $8 \%$ pour ses deux actionnaires : l'État et la CDC.

Ainsi, les responsables de ce projet, mené à titre expérimental et donc suivi de très près par les acteurs de la propriété intellectuelle en France (et probablement en Europe), devront gérer des tensions paradoxales particulièrement délicates, tout en ayant très peu de points de repère. Par tensions paradoxales nous entendons la dynamique simultanée de facteurs favorables à l'imitation du modèle nord-américain et de facteurs réduisant la portée d'un tel modèle. Une partie de ces tensions est liée à l'activité elle-même (les précurseurs nord-américains ont déjà subi quelques échecs et ont dû adapter leur modèle d'affaires au fur et à mesure qu'ils accumulaient de l'expérience), mais aussi en partie aux

\footnotetext{
${ }^{28}$ Communiqué de la Présidence de la République du 27 mai 2010 sur la création du fonds d'investissement France Brevets.

${ }^{29}$ France Brevets a signé cinq partenariats de valorisation avec des institutions publiques ou privées: Institut Telecom, INRIA, Université européenne de Bretagne, une société spécialisée dans les TIC et le CNES. France Brevet devrait signer une vingtaine de partenariats d'ici à fin 2012. Source: Chantale Houzelle, « France Brevets va valoriser les technologies du CNES », Les Echos.fr, 21 février 2012.

${ }^{30}$ Communiqué de presse du 9 juin 2011 du Ministère de la Recherche et de l'Enseignement Supérieur, à l'occasion du lancement de France Brevets.

${ }^{31}$ Depuis sa création en 2000, les stratégies juridiques d'Intellectual Venture (accord de licence, royalties, procès) ont permis de générer près de 2 milliards de dollars. Voir : Jacques Cheminat, « Un ancien CTO de Microsoft attaque Symantec, McAfee, Hynix pour violation de brevets », Le Monde Informatique, 9 décembre 2010.
} 
différences fondamentales entre le contexte dans lequel sont nés ces précurseurs et celui de France Brevets. Ce fonds d'investissement est ainsi soumis à des demandes paradoxales de la part de l'État : il doit développer la valorisation de la recherche publique et celle des PME avec pour but d'aider au développement économique mais avec pour contraintes de s'autofinancer, tout en s'interdisant les pratiques de licensing les plus efficaces sur le plan financier, le tout avec des moyens suffisants pour mériter l'attention des acteurs de la valorisation mais limités au regard des objectifs fixés.

\section{Vers un modèle hybride de politique publique en matière de propriété intellectuelle}

Nous allons tout d'abord défendre l'idée que la nature hybride de France Brevets est à l'image des objectifs supposés de son principal initiateur, la Caisse des Dépôts et Consignations (CDC) mais aussi des objectifs poursuivis par les pouvoirs publics français en matière de recherche et d'innovation : mettre les organisations publiques de recherche au service de la compétitivité de l'économie nationale. Dans ce contexte, nous montrerons ensuite que l'exemple nord-américain propose des modèles d'action en matière d'innovation et de recherche qui combinent de puissants facteurs d'attraction et de répulsion pour les acteurs publics français. La transposition du modèle nord-américain de gestion de la propriété intellectuelle au contexte français et européen ne peut donc pas se faire sans de sérieuses adaptations, pour peu qu'elle soit souhaitable.

\subsection{Un modèle hybride}

Le projet France Brevets est issu d'une initiative propre à la CDC, et qui s'inscrit pleinement dans la politique de cet établissement public dont la finalité est l'investissement à long terme et le soutien au développement des entreprises. La démarche de la CDC relève d'une vision innovatrice, voire exploratoire, qui consiste à introduire en France des dispositifs de l'économie de la connaissance mais qui ont été préalablement développés par des entreprises américaines, notamment Ocean Tomo et Intellectual Ventures. S'il s'agit effectivement de pallier les insuffisances des organismes de valorisation de la recherche publique en France, l'initiative France Brevets peut également être interprétée comme une démarche proactive permettant à la CDC de se positionner sur un marché européen émergent des brevets. Elle peut ainsi être interprétée comme une démarche opportuniste d'une structure publique (mais aux activités mixtes publiques/ privées), pour s'impliquer fortement dans un champ où elle était peu présente alors qu'il représente des enjeux économiques et politiques croissants : la valorisation de la recherche à travers la gestion de la propriété intellectuelle.

La CDC a ainsi obtenu l'engagement de l'État sur un montant de 50 millions d'euros, via le fonds de valorisation de la recherche, adopté dans le programme « Investissements d'Avenir ». Un expert en propriété intellectuelle interprète ainsi la démarche de la $\mathrm{CDC}$ : «L'idée vient de la CDC : elle a mené son projet et pris soin de déminer tous les obstacles. Elle a fait un lobbying en créant un groupe "prospective » avec des entreprises, en expliquant à l'INPI ce qu'elle voulait faire et en suscitant le rapport du Conseil d'Analyse Économique ». Même si l'emploi du concept de «lobbying» 
pour qualifier les actions de la CDC dans la création de France Brevets est à nuancer, il semble effectivement qu'elle ait réussi à orienter les décisions publiques dans un sens favorable à sa propre vision de l'organisation du marché des brevets en France. Le rapport de la commission «Juppé-Rocard» invitait les pouvoirs publics à constituer un fonds national de valorisation des projets innovants (phase de maturation des innovations et accompagnement au dépôt de brevets), doté d'un capital d'un milliard d'euros mais ne préconisait pas formellement la constitution d'un fonds d'exploitation des brevets. Ce fonds de valorisation a été mis en place dans le cadre du programme «Investissements d'Avenir » et le projet France Brevets a été greffé à ce programme en prélevant une dotation de 50 millions d'euros de l'État sur le fond d'un milliard d'euros destinés aux SATT.

Par ailleurs, le rapport du Conseil d'Analyse Économique (Guellec et al., 2010) semble avoir été «suscité » par la CDC comme nous l'ont souligné plusieurs de nos interlocuteurs (conseils en propriété intellectuelle). Pour autant, les conclusions du rapport demeurent prudentes quant aux approches fondées sur la «marchandisation » des brevets ${ }^{32}$. Les experts ayant participé à la rédaction du rapport recommandent néanmoins la création de plusieurs fonds publics de brevets «d'envergure européenne et disposant de fonds importants » qui auraient pour finalités «la maîtrise de filières technologiques clés, le soutien à l'innovation des PME et la participation à la valorisation de la recherche publique» (p. 244). La réalité de France Brevets est bien plus modeste. Doit-on y voir un échec de la CDC dans sa capacité à infléchir la politique d'investissement de l'État dans la valorisation de la recherche (et obtenir un engagement financier plus important), ou, au contraire, un succès relatif compte tenu de la prise de risque de l'État dans une opération exploratoire et dont l'utilité publique peut être questionnée? En effet, avec un capital de 100 millions d'euros, France Brevets ne possède vraisemblablement pas la surface financière critique pour peser sur telle ou telle filière technologique, ni sur la valorisation de la recherche publique. Dans ces conditions, la finalité du fonds est hybride car elle relève au moins autant d'une logique privée (prise de risque, positionnement sur le marché européen des brevets, objectifs de rentabilité des fonds investis) que d'une logique d'intérêt général au service de la mise en œuvre de la «Stratégie nationale de recherche et d'innovation ».

\subsection{Le signal du nouveau rôle de l'État en matière de politique d'innovation}

France Brevets envoie aussi un signal supplémentaire aux acteurs de la recherche et de l'innovation sur l'intérêt croissant de l'État en la matière. Alors que traditionnellement les responsables politiques s'impliquaient peu dans les questions de la propriété intellectuelle ${ }^{33}$ jugées trop techniques, cette initiative met l'accent sur le rôle des brevets dans la construction d'avantages concurrentiels durables (notamment à travers les clusters ou les grappes de brevets) mais aussi sur les limites culturelles et structurelles de la valorisation

\footnotetext{
32 Le rapport souligne ainsi que «le souci de «monétiser » la propriété intellectuelle ne doit pas prendre le pas sur d'autres considérations d'intérêt général » (p. 169).

${ }^{33}$ Par exemple, un de nos interlocuteurs, expert en propriété intellectuelle, a insisté sur le manque d'intérêt traditionnel des pouvoirs publics français pour les questions de propriété intellectuelle.
} 
de la recherche publique en France ${ }^{34}$. Elle met aussi en exergue la pression croissante que ces mêmes responsables politiques font peser sur les opérateurs publics pour démontrer leur «performance » sans toujours que cette dernière soit définie de façon satisfaisante. L'initiative France Brevets illustre également une certaine maturité dans la façon d'appréhender l'importation de modèles étrangers pour tenter de les adapter au contexte national dans un cadre expérimental, voire même de les analyser pour mieux s'en affranchir et construire un système original.

Les initiateurs de France Brevets ont clairement conscience du besoin de créer un système qui capitalise sur les enseignements nord-américains mais qui ne doit pas produire les mêmes effets pervers. France Brevets se trouve ainsi au centre de tensions paradoxales suscitées par le contraste entre ses fondements idéologiques, théoriques et institutionnels résolument néolibéraux (le recours au Marché constitue la solution aux problèmes) et sa structure et ses missions publiques. Ce sont probablement ses modalités de mise en œuvre qui lui permettent de trouver un relatif équilibre entre ces deux antagonismes apparents.

Dans la phase initiale de création de France Brevets, des représentants d'Ocean Tomo ont été associés à des groupes de travail car cette entreprise disposait d'une expertise reconnue dans le domaine des marchés de brevet qui ne pouvait qu'intéresser, au moins dans un premier temps, les initiateurs de ce dispositif. En effet, France Brevets ne constitue que le premier élément expérimental d'un hypothétique système européen facilitant les transactions sur les brevets. La présence dans sa phase initiale de représentants d'un des symboles du « modèle américain » dans ce domaine n'est donc pas surprenante. Toutefois, cette entreprise symbolise aussi certains excès, voire les dérives, du système nord-américain : selon l'un de nos interlocuteurs (expert en propriété intellectuelle) « le modèle d'Ocean Tomo est tout de même basé sur les approches de Chesbrough ${ }^{35}$ qui proposait, avant la crise, de s'inspirer du système des « subprimes » pour les appliquer au marché des brevets ». Les pouvoirs publics français cherchent ainsi à pallier la faiblesse des initiatives privées en matière de valorisation de la propriété intellectuelle en créant une structure publique qui assurera cette mission dans le cadre de la «stratégie nationale de recherche et d'innovation ». Son modèle de référence, dans son principe, est le modèle nord-américain privilégiant une approche de marché. Ses modalités d'action doivent puiser dans l'exemple d'efficience supposée des entreprises nord-américaines intervenant dans ce domaine mais sans toutefois adopter leurs modèles d'affaires agressifs. Le défi est de taille car il n'est pas aisé de combiner efficience financière et respect de commandes politiques, lesquelles sont par nature souvent fluctuantes et relevant d'une rationalité d'une nature parfois différente.

Toutefois, des questions demeurent : si les acteurs privés ne sont pas présents dans ce type d'activité, est-ce à dire que le risque est trop important au regard des intérêts concurrentiels ou économiques potentiels? L'environnement institutionnel et culturel français

\footnotetext{
${ }^{34}$ "Selon Valérie Pécresse, il existe un important décalage entre la $6^{e}$ place de la recherche française dans le monde par le nombre de ses publications et sa « valorisation » en termes de dépôt de brevets Les raisons sont multiples. Elles vont de l'insuffisante culture d'entrepreneuriat des chercheurs français, pour qui la valorisation marchande de leurs travaux n'est pas une priorité, à l'émiettement des structures, en passant par l'absence de brevet unique européen ou celle de puissants fonds de brevets capables de rivaliser avec les géants américains ». Source : Christophe Alix, « Pécresse pousse les chercheurs aux brevets », Libération, 29 juillet 2010. 35 Henry Chesbrough est professeur à l'université de Berkeley. Il a posé les principes de l'open innovation (innovation ouverte ou distribuée).
} 
est-il si défavorable à la prise de risques de la part d'investisseurs privés dans le domaine de l'exploitation de la propriété intellectuelle? Au regard de l'exemple nord-américain, est-il préférable pour l'État de faire évoluer cet environnement pour susciter les initiatives privées ou de s'y substituer tout en s'en inspirant?

\section{Conclusion}

L'initiative France Brevets s'inscrit dans un ensemble de mesures favorables à la recherche, à la valorisation de la recherche et à l'innovation. France Brevets et les « Investissements d'avenir » s'inscrivent dans une dynamique de renforcement du rôle des États qui a suivi la crise de $2008^{36}$. Ainsi, le projet France Brevets peut être interprété comme le signe d'un retour de l'État entrepreneur, palliant les insuffisances du marché par des projets structurants et de long terme (Barreau, 1990). Cela peut également être vu comme une démarche proactive pour améliorer l'efficience de la recherche publique française au service de l'économie nationale.

Nous proposons néanmoins une lecture plus nuancée, voire plus critique, de ce dispositif. La multiplication des initiatives et des programmes (pôles de compétitivité, agence nationale de la recherche, Sociétés d'Accélération de Transfert Technologique, Pôles de Recherche et d'Enseignement Supérieur (PRES)...) risque de se traduire par un émiettement des budgets consacrés à la valorisation de la recherche si ceux-ci devaient à l'avenir être réduits. Elle se traduit aussi, pour partie, par une juxtaposition de structures dont les finalités peuvent être en concurrence et donc conduire, in fine, à une inefficience globale des dispositifs. Ainsi, la convention portant création de France Brevets prévoit formellement une coopération avec les SATT qui elles-mêmes devront coordonner les actions de valorisation des autres structures de valorisation existantes.

La création de France Brevets, mais aussi des SATT, risque ainsi de rajouter à la complexité du système et à la confusion des rôles. Comme le souligne Flesia (2006), «notre pays est marqué par la coexistence d'organismes nombreux et divers (universités, grands organismes de recherche, écoles d'ingénieurs, pour nous limiter aux principaux) dont les missions, les statuts, les tutelles et les pratiques reflètent des réalités extrêmement variées et traversent pratiquement tous les domaines scientifiques et techniques ». Pour certains de nos interlocuteurs, l'action de l'État aurait pu être orientée vers le renforcement des structures existantes (augmentation des moyens alloués) ou la redéfinition de leurs missions (comme celles des PRES), de manière à atteindre les tailles critiques nécessaires.

Même si elle n'est qu'expérimentale et demeure modeste dans son envergure, l'initiative France Brevets reflète une évolution du référentiel de l'action publique (Jobert, 2005) focalisée sur la recherche d'une performance mesurable pour constituer un objectif acceptable. Dans ce contexte, où fins et moyens se trouvent inversés, le brevet devient l'un des principaux indicateurs de la performance des organisations publiques de recherche et de la capacité d'innovation des entreprises françaises. Ainsi, l'un de nos interlocuteurs relève que «Valérie Pécresse a fixé comme objectif que la moitié des brevets des 50 premiers déposants français viennent de la recherche publique. L'État cherche à être proactif dans ces domaines (...) Pour l'État français,

${ }^{36}$ Voir le rapport de la commission « Juppé-Rocard » (p. 4). 
il faut accroître la visibilité des brevets. La notion quantitative (nombre de brevets) est censée conduire à s'intéresser à la propriété intellectuelle et donc à l'innovation » (expert en propriété intellectuelle). Cette remarque est confirmée par une déclaration de la ministre ${ }^{37}$ et par les objectifs attribués aux organisations publiques de recherche. Cette approche conforte une vision quantitative qui peut se traduire par des politiques publiques sous-estimant la complexité des champs de la recherche et de l'innovation (Lallement, 2008). Pourtant, les économistes ont montré depuis longtemps que le brevet était un indicateur très imparfait de la performance en matière d'innovation : il est donc dangereux d'appliquer un tel indicateur de performance en France comme cela a été d'ailleurs souligné dans le rapport Guellec (2010).

Quel serait alors le sens de ces initiatives, du point de vue de l'action publique, si elles ne permettent pas d'accroître son efficacité globale? Il nous apparaît que ces initiatives permettent en premier lieu de créer des dispositifs valorisables dans les champs politiques et médiatiques. Ainsi, au-delà des effets d'annonce, la mise en place d'une structure comme France Brevets incarne à court terme les efforts du gouvernement en faveur de la compétitivité nationale et de l'efficacité de la recherche publique. Or, «le temps de l'innovation et de la recherche n'est pas celui de la politique » (expert en propriété intellectuelle) et ces initiatives relèveraient plus de la «cosmétique politique » que d'un plan de développement à long terme de la recherche et de l'innovation en France. Privilégiant une vision à court terme, ces initiatives n'en seraient pas moins soumises à des jeux d'acteurs qui, par opportunisme, en fragilisent la portée pour l'intérêt général. Cela rejoint la vision de Guellec (2001) pour qui «de composante de la politique de souveraineté de l'État, la politique d'innovation est devenue composante d'une politique des entreprises et des marchés ».

Nous pouvons toutefois conclure de manière moins pessimiste quant au rôle de l'État dans la définition et la mise en œuvre d'une politique publique en faveur de la valorisation de la recherche publique et de l'innovation. Le cas France Brevets renvoie plutôt à une démarche publique pragmatique car, selon les termes de Jonah Levy $(2006)^{38}$, l'État est désormais «plus à l'aise dans une position d'accompagnateur du marché (market-supporting) que dans sa position traditionnelle de pilote du marché (market-steering)». Avec France Brevets, l'État se positionne comme accompagnateur, ou facilitateur, d'un « marché » de la recherche, publique et privée, en pleine mutation. Il fait ainsi acte de sa « conversion » à une marchandisation accrue de la production de connaissances, considérée encore par beaucoup d'acteurs comme un bien public. L'État se positionne aussi comme «pilote» dans une perspective de reconfiguration d'une économie de la connaissance à l'échelle européenne. Les récentes évolutions concernant la mise en place d'un brevet unitaire européen poseront vraisemblablement, à l'échelle européenne, la question d'un marché des brevets. L'expérience France Brevets, dans son processus comme dans son contenu, permettra sûrement à la France de pouvoir tenir une position de leadership dans les débats européens. Mais au service de quelle cause : celle du Marché ou celle de la Régulation?

\footnotetext{
${ }^{37}$ Discours à l'occasion de la clôture de cérémonie de signature par France Brevets des conventions de partenariat avec I'Institut National de Propriété Industrielle (INPI), OSEO et I'Institut Télécom le 9 juin 2011. ${ }^{38}$ Cité par Borraz et Guiraudon (2008: 23).
} 


\section{Bibliographie}

ADNOT P., (2006), Rapport d'information $n^{\circ} 341$ fait au nom de la commission des Finances, du contrôle budgétaire et des comptes économiques de la Nation sur la valorisation de la recherche dans les universités, Sénat, Annexe au procès-verbal de la séance du 10 mai 2006.

BACH L., LLERRENA P., coord., (2008). Les activités de recherche contractuelle et de transfert de technologie dans les établissements français d'enseignement supérieur, rapport de recherche du bureau d'économie théorique et appliquée (BETA). Université Louis Pasteur Strasbourg, pour le Ministère de l'Enseignement Supérieur et de la Recherche.

BARREAU J., (1990). L'État entrepreneur, L'Harmattan, Paris.

BORRAZ O., GUIRAUDON V., (2008). Introduction : comprendre les évolutions de l'action publique In O. Borraz et V. Guiraudon, Politiques publiques, Presses de Sciences Po, 11-26.

CORBEL P., CHOMIENNE H., SERFATI C., (2011). L'appropriation du savoir entre laboratoires publics et entreprises : la gestion des tensions paradoxales au sein d'un pôle de compétitivité, Revue Française de Gestion 210, 149-163.

DASGUPTA P., DAVID P.A., (1994). Toward a New Economics of Science, Policy Research 23, 487-521.

DIESSEL B.H., (2007). Trolling for Trolls: The Pitfalls of the Emerging Market Competition Requirement for Permanent Injunctions in Patent Cases Post-eBay, Michigan Law Review 106, 305-345.

EMERY Y., GIAUQUE D., (2008). Repenser la gestion publique : bilan et perspective en Suisse, PPUR, Lausanne.

FLESIA E., (2006). Valorisation de la recherche, innovation et création d'entreprises, Géographie, Économie, Société 8 (1), 149-158.

FLIGSTEIN N.,(2001). The Architecture of Markets: an Economic Sociology of Twenty-First-Century Capitalist Societies, Princeton University Press, Princeton.

GUELLEC D., (2001). Les politiques de soutien à l'innovation technologique à l'aune de la théorie économique, Économie \& prévision, 150 (4), 95-105.

GUELLEC et al., (2010). Les marchés de brevets dans l'économie de la connaissance, Rapport pour le Conseil d'Analyse Économique.

GUILLAUME H., coord., (2007). Rapport sur la valorisation de la recherche, Inspection générale des finances, Inspection générale de l'administration de l'éducation nationale et de la recherche.

HUANG K.G., MURRAY F.E., (2009). Does Patent Strategy Shape the Long-Run Supply of Public Knowledge? Evidence from Human Genetics, Academy of Management Journal 52 (6), 1193-1221.

JOBERT B., (2005). Une approche dialectique des politiques publiques. L'héritage de l'État en action, Pôle Sud $21(2), 43-54$.

KEGELS C., (2009). La politique d'innovation dans une économie de la connaissance, Reflets et perspectives de la vie économique XLVIII (1), 151-159.

KENNEY M., PATTON D., (2009). Reconsidering the Bayh-Dole Act and the Current University Invention Ownership Model, Research Policy 38, 1407-1422.

KORTUM S., LERNER J., (1999). What is Behind the Recent Surge in Patenting? Research Policy 28, 1-22.

LALLEMENT R., (2008). Politique des brevets : l'enjeu central de la qualité, face à l'évolution des pratiques, Horizons stratégiques 7, janvier-mars 2008 (www.strategie.gouv.fr).

LESOURNE J., RANDET D. (2010). La recherche et l'innovation en France, Odile Jacob, Paris.

LEVY J., (ed.) (2006). The State after Statism. New State Activities in the Age of Liberalization, Harvard University Press, Cambridge.

LÉVY M. et JOUYET J.P., (2006). L'économie de l'immatériel - la croissance de demain, rapport de la commission sur l'économie de l'immatériel, Ministère de l'Économie, des Finances et de l'Industrie, Paris.

MARTINET A.-C., (1990). Épistémologie de la stratégie In Martinet, A.-C., Épistémologies et Sciences de Gestion, Economica, 211-236. 
MCCURDY D., (2008). Patent Trolls Érode the Foundation of the U.S. Patent System, Science Progress, fallwinter 2008/2009, 78-86.

MOWERY D.C., NELSON, R.R., SAMPAT, B.N., ZIEDONIS, A.A., (2001). The Growth of Patenting and Licensing by U.S. Universities: an Assessment of the Effects of the Bayh-Dole Act of 1980, Research Policy 30, 99-119.

MULLER P., (2005). Esquisse d'une théorie du changement dans l'action publique : structures, acteurs et cadres cognitifs, Revue française de science politique 55 (1), 155-187.

NATIONAL RESEARCH COUNCIL, (2010). Managing University Intellectual Property in the Public Interest, Committee on Management of University Intellectual Property: Lessons from a Generation of Experience, Research and Dialogue.

PÉNIN J., (2010). Le problème des «patent trolls » : comment limiter la spéculation sur la propriété intellectuelle dans une économie fondée sur la connaissance, Innovations : Cahiers d'économie de l'innovation 32, 35-53.

POLlitT C., BOUCKAERT G., (2004)., Public Management Reform - A Comparative Analysis, Oxford University Press, Oxford.

PORTER M. E., (1998). Clusters and the New Economics of Competition, Harvard Business Review, November December 1998, 77-90.

REITZIG M., (2004). The Private Values of "Thickets" and "Fences": Towards an updated Picture of the Use of Patents across Industries, Econ. Innov. New Techn. 13 (5), 457-476.

REITZIG M., HENKEL J., HEATH C., (2007). On Sharks, Trolls, and Their Patent Prey - Unrealistic Damage Awards and Firms' Strategies of "being infringed", Research Policy 36, 134-154.

TEECE D.J., (1998). Capturing Value from Knowledge Assets: The New Economy, Markets for Know-How, and Intangible Assets, California Management Review 40 (3), 55-79.

TIXIER J., CASTRO GONÇALVES L., (2008). Les pôles de compétitivité à l'heure de l'évaluation : quel modèle de cluster à la française ? Réalités industrielles mai, 103-110. 
